\title{
Aportes de la teoría psicoanalítica para la comprensión de las adicciones ${ }^{1}$ \\ Contributions of psychoanalytic theory for the understanding of addictions
}

Recibido: 27 de junio de 2016 - Revisado: 15 de mayo de 2017 - Aceptado: 23 de julio de 2017.

Miguel Gutiérrez-Peláez ${ }^{2}$

Laura Andrea Blanco-González ${ }^{3}$

Carlos Márquez ${ }^{4}$

\section{Resumen}

El presente artículo contribuye a la comprensión de la problemática de las adicciones a partir de los planteamientos teóricos del psicoanálisis. Para ello, se revisaron las posiciones del psicoanálisis freudiano, lacaniano y otras corrientes psicoanalíticas respondiendo a las siguientes preguntas de investigación: ¿Cómo se comprenden las adicciones desde un marco de referencia psicoanalítico?, ¿Cómo desde la teoría freudiana y la teoría lacaniana se aborda la comprensión de la adicción?, ¿Qué plantean otras corrientes del psicoanálisis sobre ésta problemática? Se encontró que es un campo de constante publicación de literatura psicoanalítica, que es un fenómeno clínico en aumento y que se requiere de investigación continua.

\section{Palabras clave}

Adicción, goce, psicoanálisis, toxicomanías, teoría lacaniana, Freud.

\begin{abstract}
The following article contributes to the understanding of the problem of addictions from a psychoanalytic point of view. The different theoretical positions of Freudian, Lacanian and other psychoanalytic schools were reviewed in order to answer the following research questions: How are addictions understood from a psychoanalytic point of view? How from Lacanian and Freudian theory - is the understanding of addiction addressed? What do other schools of psychoanalysis state about this problem? It is found that it is a field of constant publication in the psychoanalytic literature that it is an increasing clinical phenomenon and that continuous investigation is required in this field.
\end{abstract}

\section{Keywords}

Addiction, jouissance, psychoanalysis, toxicomany, lacanian theory, Freud.

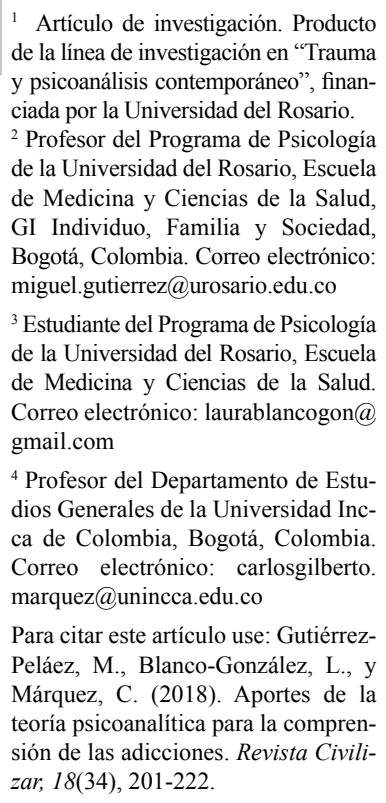

Artículo de investigación. Producto de la línea de investigación en "Trauma y psicoanálisis contemporáneo", financiada por la Universidad del Rosario. ${ }^{2}$ Profesor del Programa de Psicología de la Universidad del Rosario, Escuela de Medicina y Ciencias de la Salud, GI Individuo, Familia y Sociedad, Bogotá, Colombia. Correo electrónico: miguel.gutierrez@urosario.edu.co

${ }^{3}$ Estudiante del Programa de Psicología de la Universidad del Rosario, Escuela de Medicina y Ciencias de la Salud. Correo electrónico: laurablancogon@ gmail.com

${ }^{4}$ Profesor del Departamento de Estudios Generales de la Universidad Incca de Colombia, Bogotá, Colombia. Correo electrónico: carlosgilberto. marquez@unincca.edu.co

Para citar este artículo use: GutiérrezPeláez, M., Blanco-González, L., y Márquez, C. (2018). Aportes de la teoría psicoanalítica para la comprensión de las adicciones. Revista Civilizar, 18(34), 201-222. 


\section{Introducción}

En Colombia, el uso y abuso de sustancias tóxicas es un tema de debate permanente por sus repercusiones a nivel social, psicológico, económico y político y por su manejo en las instancias judiciales y en el sector de la salud. En los últimos años el Gobierno Nacional ha realizado diversos estudios para estimar la magnitud del consumo de drogas en el país, actualizar el conocimiento sobre la problemática, establecer la percepción social de riesgo asociado y estimar la oferta de drogas a la población.

Cotejando los dos últimos se observa un aumento en el consumo de sustancias psicoactivas entre los años 2008 y 2013. Las problemáticas psicosociales que se derivan de ello han prendido las alarmas del sector de la salud y hacen pertinente revisar los aportes a la comprensión y a los posibles tratamientos para los sujetos afectados por esta problemática. El psicoanálisis, desde los escritos de Sigmund Freud hasta nuestros días, ha hecho aportes teóricos y clínicos para la comprensión del fenómeno de la adicción.

Las adicciones son un fenómeno que incumbe a los países por las dimensiones geopolíticas que alcanza; a la iglesia, a las comunidades y a las organizaciones internacionales como la Organización de las Naciones Unidas (ONU) y la Organización Mundial de la Salud (OMS), por sus efectos a nivel mental, psicológico y socio-cultural, entre otros muchos (Requiz, 2000). Por ello hay una permanente discusión sobre las repercusiones económicas y legales de la distribución y el consumo.

La dependencia de sustancias químicas es un factor que puede influir en patrones de conducta violenta y es responsable de gastos significativos en el sistema económico contemporáneo. A pesar de que en la historia el uso de drogas ha sido un fenómeno recurrente, desde los fines medicinales hasta los rituales o la recreación (como puede verse desde algunos pergaminos del Corán y del Mar Muerto hasta en jeroglíficos del antiguo Egipto), la psicopatología derivada del consumo de sustancias psicoactivas es un fenómeno relativamente nuevo (Fine y Juni, 2001; Naparstek, 2014).

\section{Método}

La presente investigación hace una revisión de las bases de datos PepWeb, Ebsco, y Jstor y analiza los aportes teóricos para la comprensión del fenómeno de las adicciones, iniciando desde las posiciones del fundador de la disciplina, Sigmund Freud y pasando por Jacques Lacan y otros autores de la disciplina psicoanalítica. La finalidad será responder a las siguientes preguntas de investigación: ¿Cómo se comprenden las adicciones desde un marco de referencia psicoanalítico?, ¿Cómo desde la teoría freudiana y la teoría lacaniana se aborda el conocimiento sobre la adicción?, ¿Qué plantean otras corrientes del psicoanálisis sobre ésta problemática? Dado que gran parte de las publicaciones psicoanalíticas se realizan en libros y en editoriales especializadas por fuera de estas bases de datos indexadas, se consultaron además publicaciones representativas y reconocidas en este campo. Adicionalmente, nos apoyamos en la exhaustiva recensión de la bibliografía psicoanalítica realizada por Héctor López (2007).

La estructura argumentativa del artículo será la siguiente: se demostrará que lo que distingue la orientación por lo real en el abordaje de las adicciones es el abandono en lo teórico de la pretensión de establecer universales a partir de los fenómenos particulares, acompañado de un acento puesto en el uso singular que cada sujeto hace del tóxico, extrayendo las enseñanzas que cada caso produce a partir de una reflexión permanente sobre la experiencia clínica. Esto desemboca en el principio de que el tóxico separa al sujeto del goce fálico y de esta manera lo aleja del sufrimiento concomitante con las dificultades que plantea el deseo para cada sujeto. 
Para ello, partiremos de que la propuesta psicoanalítica en general es que las adicciones no constituyen una enfermedad, sino un asunto que atañe éticamente al sujeto tomado en su especificidad. Luego, transitaremos por el aporte de Sigmund Freud a esta problemática ya que, para él, no se trata en la adicción de la búsqueda de la felicidad, sino de la evitación del displacer. La función de la droga se entiende como un "quita penas". En la obra de Lacan, partiendo de los argumentos de Freud, encontraremos también la droga como un laberinto sin salida para los avatares del deseo humano.

En contraste, desde otras orientaciones psicoanalíticas, se intenta captar la esencia común de los múltiples fenómenos de la toxicomanía a partir de explicaciones de carácter universal. Reconocemos allí un gesto inductivo, que tiende al modo científico de aproximación de los fenómenos, y que se distingue netamente de la orientación por lo real.

\section{El lugar del psicoanálisis en los abordajes clínicos contemporáneos de las adicciones}

La propuesta psicoanalítica es que las adicciones no constituyen una enfermedad, sino un asunto que atañe éticamente al sujeto tomado en su especificidad. Sin embargo, para su tratamiento es más común que se recurra a terapias cognitivo-conductuales, terapias biológicas, organizaciones como Alcohólicos Anónimos (A.A.) o Narcóticos Anónimos (N.A.) o a terapias humanistas centradas en el cliente. Esto se debe a que el psicoanálisis se distingue del tratamiento médico de esta problemática, que constituye un intento de "fundamentar el estatus de enfermedad de la adicción" (López, 2011, p. 42).

El psicoanálisis, desde sus inicios, ha planteado que hay que centrarse en los conflictos intra-psíquicos que sostienen la adicción más que en la sustancia misma. Según Ferenczi (1911), "la acción unilateral agitadora de los partidarios del antialcoholismo intenta ocultar el hecho de que, en la gran mayoría de los casos, el alcoholismo es una consecuencia [...] de la neurosis, pero no su causa" (p.106; citado por López, 2011, p.42; Gutiérrez-Peláez, 2012). Bleuler (1911) al respecto suponía que "sabemos apenas más de la relación entre el alcohol y nuestra vida de complejos, que el público que atribuye el alcoholismo de un hombre a la maldad de su mujer [...].” (p. 852; citado por López, 2011, pp. 45-46).

Desde entonces el fenómeno de la adicción fue un tema de gran interés para varios autores psicoanalíticos. Rado (1926), al introducir el término de "metaerotismo", propone que en la adicción hay un ataque a la organización sexual biológica en donde "se deja de lado a todo aparato sexual periférico [...] y se permite que los estímulos excitantes operen directamente sobre el órgano central" (p. 38), produciéndose una independencia de los modos habituales de gratificación y del funcionamiento psíquico en general. Más adelante, hacia 1933, plantea que en la adicción se instaura un "régimen farmacotímico" el cual "restringe [...] la libertad de acción del yo" (p. 79). Esta concepción en donde los determinantes inconscientes se independizan del modo habitual de funcionamiento llevó al retiro total de la sustancia tóxica como primera medida en el tratamiento de una adicción, acompañada de un trabajo terapéutico (López, 2011).

Glover (1928) define la adicción como una "seudoperversión" en donde la sustancia sustituye la sexualidad, idea que tiene resonancias con el planteamiento freudiano según el cual la masturbación era la primera adicción de la cual se desprendían las demás. Según Glover, los tipos de adicción representan variaciones en los mecanismos empleados para controlar la excitación, por medio del control de las cargas sádicas, la droga queda reducida a un objeto parcial y, dependiendo de "si predomina un aspecto sádico o uno reparador, la adicción sería más nociva o inocua" (López, 2011, p. 44). 
El consumo adictivo "queda referido a una estructura psíquica pre-mórbida en donde el lugar privilegiado que ocupa la sustancia en la economía psíquica [se hace] equivalente al del objeto fetiche" (p. 44). Quizá la idea más crucial en su teoría sobre la toxicomanía, y la razón por la cual la detención del consumo es reemplazada por el método psicoanalítico tradicional, es que sería imposible curar una adicción a menos que se estudien sus causas subyacentes: "la dependencia a una sustancia es en gran parte psicológica; el psicoanalista exige la suspensión de todo juicio moral sobre la adicción a la droga" (p. 45). De los planteamientos de Glover se deriva el que el tratamiento psicoanalítico se centre en los mecanismos inconscientes de la adicción y no en la sustancia como tal.

Como hemos visto, existe una discordancia entre esta concepción del psicoanálisis y el esfuerzo de la medicina por hacer de la adicción una enfermedad. Desde el punto de vista histórico es por lo menos curioso que, aunque el consumo de alcohol u otras sustancias psicoactivas siempre han representado un problema para la sociedad, no fue sino hasta comienzos del siglo XIX que comienza a considerarse como una enfermedad.

En 1849, Magnus Huss empleó por primera vez el término alcoholismo en Alcoholismus Chonicus identificando varios desórdenes asociados al consumo de alcohol (Rivers y Shore, 1997). Después, el concepto de dependencia fue extendido a otras sustancias tóxicas adictivas y empieza a generarse una real preocupación por la detención del consumo y el tratamiento de la adicción. Así se establece el consumo crónico de sustancias psicoactivas como una enfermedad. Al respecto Jellinek (1960) destaca las alteraciones del funcionamiento celular, el aumento de la tolerancia del tejido, la falta de control y la incapacidad para detenerse como sus características.

En 1992 el Joint Committee of the National Council on Alcoholism and Drug Depen- dence and the American Society of Addiction Medicine publica un artículo que define el alcoholismo como una enfermedad primaria, "crónica, con factores genéticos, psicosociales y ambientales que influencian su desarrollo y sus manifestaciones $[. .$.$] muchas veces progresiva$ y fatal. Se caracteriza por falta de control sobre el consumo, intereses centrados en la droga alcohol, uso de alcohol a pesar de las consecuencias adversas, y distorsiones en el pensamiento, especialmente, la negación" (Morse y Flavin, 1992, p. 1013). Con esta definición se entiende que el sujeto tiene una discapacidad y por tanto "no dispensa de responsabilidad en el sentido legal" (López, 2011, p. 48). López (2007) resalta la paradoja de que por un lado la adicción sea un acto ilegal que debe ser procesado y judicializado, por el otro, que el sujeto que consume sea un enfermo y requiera de tratamiento.

Actualmente en la quinta versión del Diagnostic and Statistical Manual of Mental Disorders (DSM-V), la dependencia a sustancias implica tolerancia, abstinencia, falta de control sobre el consumo y la constante búsqueda del consumo a pesar de sus consecuencias, una línea de pensamiento acorde con el planteamiento inicial de Jellinek. El National Institute on Drug Abuse -NIDAI por sus siglas en inglés- define la adicción como "una enfermedad del cerebro crónica, con recaídas frecuentes" (López, 2011, p. 49). Estas definiciones se mantienen en un nivel descriptivo, de modo que este intento de hacer una integración psicoanalítica y médica sobre la perspectiva de la adicción, "sólo constata la diferencia entre las aproximaciones para luego volver a las hipótesis psicoanalíticas clásicas. En estos intentos sí se gana cierta flexibilidad en el ámbito de las propuestas terapéuticas, en el sentido de abrir la posibilidad de combinar tratamientos con orientaciones diversas" (p. 50).

Definir la adicción como una enfermedad plantea un tercer obstáculo y es la forma cómo se caracteriza este fenómeno. Según López (2011), las dificultades en la definición del fenómeno se 
hacen claras cuando se afirma que el sujeto tiende a mantener una conducta a pesar del daño que esta provoca y que, en algunas circunstancias, hay una falta de control subjetiva; el consumo de sustancias y su posterior dependencia a ellas es en sí una circunstancia subjetiva. En La sexualidad y la etiología de la neurosis (1898), Freud afirmaba que no todo sujeto que consume una sustancia desarrolla una adicción $\mathrm{y}$ esto ha sido comprobado innumerables veces en el transcurso de la experiencia clínica psicoanalítica (López, 2011).

Así, lo central en la adicción no es la conducta del consumo como tal, sino las vivencias del sujeto en relación con la sustancia. Éste siente un llamado a obtener un placer que se asocia con el consumo del tóxico que puede adquirir una intensidad suficiente de forma "éste tiende a consumir", pero su capacidad de decisión no está anulada. "Por lo tanto, es [...] posible que el sujeto decida, dependiendo de las circunstancias, decir que no al llamado y consumir controladamente o abstenerse de hacerlo" (López, 2011, p. 52). Centrar el tratamiento de la adicción en el llamado a consumir y no en la conducta como tal, mantiene una consonancia con los planteamientos clásicos de Glover y Rado en donde se privilegia la búsqueda de las causas psíquicas del consumo.

Heyman (2009) cuestiona las definiciones tradicionales del fenómeno de la adicción y precisa qué caracteriza a la sustancia toxica adictiva: 1) Es especiosa, es decir, involucra una recompensa de gran valor debido a la cualidad e intensidad de satisfacción que ésta produce. 2) No lleva un feedback auto-inhibitorio connatural al resto de las actividades, es decir, el consumo de la sustancia "no lleva a la saciedad o fatiga... que hace que su valor de recompensa relativo al resto de las alternativas de gratificación disponibles sea cada vez mayor" (p. 125).

La diferencia entre entender la adicción desde la conducta o desde la instauración del llamado a consumir tiene importantes impli- caciones en un acercamiento entre la posición médica y psicoanalítica. Sin embargo, enfatizar el llamado a consumir también representa dificultades en la observación clínica pues éste no se muestra fácilmente (López, 2011). Distintos autores como Sachs (1923), Rosenfeld (1960), McDougall (1974), Miller y Rollnick (1991) y Morse y Flavin (1992), entre otros, han abordado la forma en la que se produce la relación del individuo con el llamado o el deseo a consumir sustancias y los diferentes niveles de intensidad en que se manifiesta.

Con la creación de organizaciones como A.A. o N.A, el surgimiento de terapias biológicas y tratamientos cognitivo-conductuales, el psicoanálisis como tratamiento para la adicción ha ido quedando en un segundo plano. Sin embargo, la literatura sostiene que existen varias razones por las cuales este "representa una herramienta [...] útil tanto en el entendimiento de la adicción, como en la formulación de [...] intervenciones terapéuticas destinadas a permitir cambios profundos y duraderos en la persona" (Acuña, 2009, p. 2).

Bateman, Brown y Pedder (2000), Southwick y Satel (1990), Bateman y Holmes (1995), Kandel (1999), Kihlstrom (1987), Underwood (1996) y Epstein (1994) destacan el hecho de que el psicoanálisis le dé un lugar destacado a las emociones y al inconsciente del individuo como factor determinante de sus conductas. "Más aún, la aproximación psicodinámica permite un entendimiento holístico de cada paciente, pues considera las interrelaciones entre su biografía, su personalidad y su trastorno psiquiátrico -la adicción en este caso- el que es visto como resultado de conflictos psíquicos" (Acuña, 2009, p. 2). Este autor destaca además que el psicoanálisis es una disciplina narrativa que asume que la mente humana no es investigable por métodos científicos y que siendo una disciplina hermenéutica, debe lograr una coherencia interna que debe someter sus afirmaciones a escrutinio científico para así lograr una validación externa. Autores como Champion, 
Goodall y Rutter (1995) y Rutter (1999) han demostrado la importancia de las experiencias de la infancia y su rol en el desarrollo de psicopatologías posteriores. Igualmente, LeDoux (1992) y Van der Kolk (1996), por medio de investigaciones neuropsicológicas con víctimas de trauma psicológico concluyen, que "los recuerdos emocionales pueden fijarse sin ningún registro consciente de su incorporación como tales" (Acuña, 2009, p. 2). El psicoanálisis permite orientar la comprensión del fenómeno y el manejo de la relación paciente-terapeuta y "las dinámicas de grupo, independientemente del contexto en que éstas se presenten" (Acuña, 2009 , p. 3). Finalmente, las investigaciones con sujetos adictos han demostrado que las perturbaciones emocionales y los conflictos interpersonales son desencadenantes de las recaídas y de la ansiedad que la droga produce.

\section{Sigmund Freud y su planteamiento sobre las adicciones.}

La novedad del enfoque de Freud está en que para él la adicción no se trata de la búsqueda de la felicidad, sino en la evitación del displacer. La función de la droga se entiende como un "quita penas": "la vida [...] resulta gravosa: nos trae [...] dolores, desengaños, tareas insolubles. Para soportarla no podemos prescindir de calmantes [...] los hay, quizá de 3 clases: poderosas distracciones [...], satisfacciones sustitutivas [...] y sustancias embriagadoras que nos vuelven insensible a ella" (Freud, 1930/2010, p. 75).

Hacia 1915, Freud define la pulsión como una fuerza constante que induce en el aparato psíquico la necesidad de hacer un trabajo para cancelarla mediante una satisfacción, que hay que entender como una "modificación apropiada a la meta de la fuente interior del estímulo" (Freud, 1915/1996, p. 114).

Para lidiar con el "dolor de existir" o la insatisfacción del deseo, el elemento más poderoso y quizás también el más perjudicial es el objeto o sustancia química. Este dolor de existir es una "pasión por evitar el dolor" que caracteriza al consumo de drogas (López, 2007). Por ello se produce la dependencia de la sustancia, que inevitablemente conlleva a más dolor.

Las primeras ideas de Freud sobre el efecto de las sustancias tóxicas se encuentran en su libro Sobre la cocaína (1884/1980), donde descubre cómo actúa la cocaína sobre las "afecciones dolorosas". Aunque en primera instancia este término se refiere al dolor físico, se irá complejizando y tendrá varios significados. Por ejemplo, la "afección dolorosa" se produce cuando hay una sobrecarga libidinal en alguna parte del cuerpo, cuando un órgano es el representante de una idea reprimida o, sencillamente, cuando hay una satisfacción aún no cumplida. Este dolor puede "experimentar una cancelación tóxica" (Freud, 1884/1980, p. 91), lo cual permite evitarlo o disminuirlo, pero no interviene sobre su causa ni permite alcanzar un goce como tal.

Más allá de estas primeras ideas sobre el efecto de las sustancias tóxicas, la primera explicación real que da Freud sobre las adicciones surge del campo de la sexualidad infantil. Él considera a la masturbación como la adicción primordial (Abraham, 1908; Gavlovski, 2011; López, 2007; López, 2006) “el acto masturbatorio, cuando deviene compulsivo, conserva una llamativa afinidad con el acto adictivo cualquiera que sea" (López, 2007, p. 21).

Como ya vimos, en La sexualidad y la etiología de las neurosis, Freud (1898) afirma que no todos los sujetos que han experimentado con sustancias tóxicas desarrollan una adicción. Si el tóxico compensa la falta de un goce sexual, en los casos en los que no es posible restablecer una vida sexual normal, una recaída es certera (López, 2007). Esta última afirmación lo lleva a formular una hipótesis que jamás abandonará: "el aparato psíquico sólo responde a la ley de la ventaja [...] sería incapaz de la renuncia a una satisfacción sino se le ofreciera a cambio una 'compensación', es decir, un 'plus de 
goce' a cambio de aquel goce directo al que se renuncia" (López, 2007, p. 24). Estas premisas freudianas tienen efectos muy concretos en la orientación que deberían tener los tratamientos clínicos para una adicción, pues no basta con privar al sujeto del objeto al cual se fijó, sino que esto debe ir acompañado de un aumento de sus satisfacciones (López, 2007).

En 1905, Freud identifica elementos similares entre la neurosis y las adicciones o toxicomanías y establece una relación entre ambas: la abstinencia de una satisfacción sexual (López, 2007). Posteriormente al publicar Dostoievski y el parricidio en 1928, ubica una "nueva adicción" que surge también de la masturbación: la adicción al juego. Freud ubica dos partes en la masturbación: la evocación de la fantasía la cual lleva a una satisfacción sexual autoerótica; y luego esta acción se fusiona con una representación optativa "perteneciente al círculo de la elección del objeto que sirve para dar [...] realidad a la situación en que tal fantasía culminaba" (Freud, 1908, p. 955). Se aclara así la creencia del adicto en que lo que causa su adicción es la búsqueda de la felicidad.

Posteriormente, en El malestar de la cultura (1930), Freud establece que lo que mueve al ser humano a actuar es esta búsqueda de la felicidad con el fin de evadir el dolor, así como experimentar sensaciones placenteras, y a este último término lo denominó "felicidad" (citado por Salomone, 2011, p. 28). Muestra así los impasses de la condición humana y la importancia de un remedio para tratarlos (Josson, 2013). Los hombres, en la búsqueda incesante de la felicidad, tienen dos fines posibles: el negativo, que consiste en evitar el dolor y el positivo cuyo fin son las sensaciones placenteras (Freud, 1930/2010).

Freud ubica al alcoholismo como una respuesta ante la imposibilidad de recorrer los caminos dispuestos por el lenguaje: "más en la edad adulta la crítica que ha reprimido el placer de disparatar llega ya a adquirir fuerzas, que no puede ser eludida [...] sin la cooperación de medios auxiliares tóxicos. El [...] servicio que el alcohol rinde al hombre es el de transformar su estado de ánimo. El buen humor surgido endógenamente o tóxicamente provocado, debilita las fuerzas coercitivas $[. .$.$] y hace accesibles$ [...] fuentes de placer. Es instructivo ver cómo conforme el buen humor va imponiendo su reinado van disminuyendo las cualidades que del chiste exigen, [...] bajo la influencia del alcohol el adulto se convierte [...] en niño, al que proporciona placer la libre disposición del curso de sus pensamientos sin observación de la coerción lógica" (Freud, 1905/1997, p. 122). Sin embargo, para él se trata de dos mecanismos de defensa distintos de los cuales el humor es más exitoso, pues se manifiesta en el lenguaje (López, 2007).

En palabras de Freud (1927): "el repudio de las exigencias de la realidad y la imposición del principio del placer aproxima el humor a los procesos regresivos [...] que nos ocupan en la psicopatología. Al rechazar la posibilidad del sufrimiento, el humor ocupa una plaza en la serie de los métodos que el aparato psíquico humano ha desarrollado para rehuir la opresión del sufrimiento [...]. El humor debe a esta vinculación una dignidad que le falta del todo, por ejemplo, al chiste, pues éste sirve tan sólo al beneficio placentero, o bien pone esta ganancia al servicio de la agresión" (Freud, 1927/2010, p. 159). Por lo tanto, el humorista triunfa donde el adicto fracasa.

\section{Las toxicomanías en la obra de Jacques Lacan}

Al igual que Freud, Jacques Lacan no elabora una teoría específica sobre las toxicomanías, pero sí dejó elementos conceptuales y clínicos de gran valor para la comprensión psicoanalítica de este fenómeno. En la relectura de los conceptos freudianos que hace Jacques Lacan, encontraremos la misma base argumentativa: La toxicomanía representa una salida en falso de los callejones sin salida tanto del deseo 
como de las satisfacciones humanas, una salida ilusoria que le promete al sujeto hacer soportable la realidad.

En 1938, Lacan escribe La Familia. Al defender la noción de complejo contra la del instinto, reconstruye el desarrollo psíquico del sujeto sobre tres estructuras sucesivas: el complejo de destete, el complejo de intrusión y el complejo de Edipo. Lacan menciona la relación existente entre las toxicomanías por vía oral y el complejo de destete, el cual se encuentra dominado por factores culturales (López, 2007). Si en el reino animal el instinto maternal cesa cuando la cría se ha desarrollado, en los seres humanos el destete ocurre por una regulación cultural. Por ello a menudo puede ser considerado como un trauma psíquico con efectos devastadores como las toxicomanías.

Se evidencia así la sintonía con la teoría inicial de Freud sobre el trauma, en donde el destete comporta contingencias operatorias. Lacan afirma que el rechazo del destete es lo que el adicto repite más adelante por medio de una ingestión tóxica, compulsiva y creciente (López, 2007), que lo sitúan en una posición de indefensión por partida doble. En primer lugar, la indefensión a la que está expuesto todo ser humano cuando lo separan del pecho de su madre. La segunda es la del adicto con respecto a la droga. "El sujeto está indefenso frente a su compulsión al ingerir el tóxico, acto acompañado por la fantasía de la posesión ilimitada del pecho materno, es decir, la renegación del destete" (López, 2007, p. 48).

Más adelante, en 1946, Lacan escribe Acerca de la causalidad psíquica. Allí, Lacan menciona nuevamente el complejo de destete y discute además el trauma en el nacimiento. En ambos textos, Lacan describe la adicción a partir del complejo de destete a raíz de un trauma no elaborado. Sin embargo, en el segundo, la forma de abordar la adicción no se limita a la pérdida del pecho materno, sino a una pérdida estructural. La teoría de Lacan no queda encap- sulada en el complejo oral, sino que "se enuncia bajo la forma de la muerte” (López, 2007, p. 53) cuya importancia se rescatará en el complejo de castración. Para él existe una salida a este complejo: "la simbolización de la pérdida mediante el juego, esto es, la transposición al lenguaje de una realidad inabordable" (López, 2007, p. 53). Una falla en este proceso conduciría al sujeto a una repetición del fracaso en lograr una identificación con el goce perdido y una de las formas en las que aparece este fracaso es precisamente la ingesta de sustancias tóxicas.

Lacan (1959) menciona nuevamente la problemática de las toxicomanías citando un artículo donde Glover afirma que la toxicomanía es una instancia de la relación perversa cuyo fin es evitar "el encuentro de una falta insoportable de la realidad" (López, 2007, p. 55). Lacan hace énfasis en el error de partir de una premisa falsa y aunque su objetivo no era centrarse en el problema de la adicción, rescata la idea de Glover de la función universal del mecanismo perverso, la renegación de la realidad que juega un rol importante en las toxicomanías.

Así, mientras que Glover "se ejercita entonces en reconstruir las inserciones cronológicas de la [función perversa], es decir, que inserta sus apariciones en un sistema de anterioridad y posterioridad, en el cual se escalonan como más primitivos los trastornos psicóticos y después vienen los trastornos psicóticos, [de manera que] [e]ntre ambos se inscribe la toxicomanía, que corresponde a una etapa intermedia" (Lacan, 1959/1970, p. 401), para Lacan, "la toxicomanía responde a un arraigo de puntos de fijación intermedia que permiten mantener una continuidad que reniega del desgarrón del yo, lo cual ya es una entrada en el campo de la perversión como cuadro clínico" (López, 2007, p. 56). Así, en la enseñanza de Lacan, la ingesta de sustancias tóxicas no se sitúa en la dialéctica simbólica, sino que "se mantiene encerrado para uso personal" (López, 2007, p. 57) y esto es inútil pues constituye el esfuerzo por un deseo sin Otro. 
Más adelante, entre 1959 y 1967, Lacan escribe la Subversión del sujeto y dialéctica del deseo en el inconsciente freudiano (1959) y Del psicoanálisis en sus relaciones con la realidad (1967). Ambos abordan la experiencia con sustancias tóxicas alucinógenas. En el primero, el interés de Lacan yacía en ejemplificar la diferencia entre una experiencia psicoanalítica y otros estados calificados por la psicología como "profundos" (López, 2007).

Su criterio [de cierta psicología] es la unidad del sujeto que es, $[\ldots]$ como si se tratase del retorno de cierto sujeto del conocimiento o como si lo psíquico tuviese que hacer valer como revistiendo al organismo [...] Ya se trate de los estados de entusiasmo en Platón, de los grados del samadhi en el budismo, o del Erlebnis, experiencia vivida de lo alucinógeno $[\ldots]$ suponemos que se está bastante informado de la praxis freudiana para darse cuenta de que semejantes estados no desempeñan en ella ningún papel (Lacan, 1959/1985, p. 775).

Lacan concluye que el tóxico no es una vía para conocer el inconsciente, pues las sustancias son un recurso cuyo fin es evitar "una verdad intolerable que sólo se dice en la palabra" (López, 2007, p. 59). La experiencia con el tóxico no es un recurso para conocer la verdad, sino que sepulta lo inconsciente. Conclusión coherente con Freud pues afirma que la intoxicación es un medio para soportar la vida, pero no para conocer el inconsciente. En el segundo texto mencionado, Lacan relata la experiencia alucinógena como un medio para demostrar lo que no es un trabajo psicoanalítico y concluye que esta intoxicación brinda una ilusión de creer que se ha alcanzado una verdad suprema o "iluminación” (López, 2007).

\section{La problemática de las adicciones en el psicoanálisis anglosajón}

Veremos ahora, cómo desde otras orientaciones, intenta captarse a partir de una serie de explicaciones de carácter universal la esencia común de los múltiples fenómenos de la adicción. Reconocemos allí un gesto inductivo, que tiende al modo científico de aproximación de los fenómenos y que, como veremos más adelante, se distingue netamente de la orientación por lo real.

Khantzian y Mack (1983) establecen que un sujeto adicto a un tóxico selecciona la sustancia de su adicción teniendo en cuenta el efecto de la sustancia y la parte afectiva que quiere medicar. Wurmser (1974), por su parte, afirma que la adicción no es más que una búsqueda de un objeto que pueda "proveer un alivio externo para una urgencia interna de un impulso abrumador" (p. 25). Dodes (1990) establece que la adicción es una consecuencia de la rabia narcisista y el resultado de una sensación de indefensión. Según Rodríguez de la Sierra (2012), un individuo consume drogas o alcohol por una dependencia psicológica poderosa que lo empuja a la sustancia para "evadir, regular o escapar de sentimientos dolorosos y angustiantes" (p. 81), idea que implica una lectura particular de la explicación de Freud sobre el consumo, en donde la droga es considerada un "quita penas". Whitman-Reymond (1988), por su parte, explora la formación de una adicción desde una pérdida temprana en el desarrollo. Este psicoanalista se ha interesado en la problemática de la adicción, particularmente en la ludopatía o juego patológico y relata la experiencia de adicción al juego de uno de sus pacientes en donde llega a la conclusión general de que "la adicción es simultáneamente el acting-out de una historia traumática, así como una defensa contra ella" (p. 145).

Dodes (2002) afirma que "cada acto adictivo es precedido por un sentimiento de indefensión e impotencia. El comportamiento adictivo funciona para reparar este sentimiento subyacente de la indefensión" (citado por Silverman, 2005, pp. 912-913). Según Dodes, no se debe ver la adicción como una debilidad, sino como una acción eficaz -aunque destructiva- que sirve para restaurar un sentimiento de poder. Así mismo, afirma que los grupos de apoyo como 
N.A o A.A. pueden ser de gran ayuda para individuos que sufren de adicción, pero cuestiona seriamente qué tan necesario y efectivo puede resultar para estas personas el declararse como "adicto" y afirmarse como indefensos ante su adicción - crítica también realizada por Réquiz (2000).

Dodesestablecelanecesidadde un enfoque terapéutico que se base en explorar la historia única y personal de cada sujeto, reconociendo que el comportamiento adictivo es una reacción a ese sentimiento de indefensión: "la adicción es un problema que yace en la persona, no en la capacidad de la droga o drogas para producir efectos físicos" (citado por Silverman, 2005, p. 914). Este autor busca alentar a los sujetos adictos a no sucumbir a los programas de rehabilitación que los representan y caracterizan como personas débiles e indefensas que carecen de fuerza de voluntad, sino, por el contrario, a recuperar el auto respeto y la autoestima que han perdido, a adquirir un entendimiento de la depresión, la auto denigración, la falta de poder y la rabia impotente que llevan dentro y que los ha llevado a su conducta adictiva, teniendo así la posibilidad de encontrar nuevas y mejores soluciones para su vida.

Igualmente, Dodes cuestiona algunos mitos sobre la adicción como, por ejemplo, la idea de que la adicción es genética o que es el resultado de un químico defectuoso en el cerebro; el consenso general de que los sujetos adictos son autodestructivos; la idea de que "hay que tocar fondo" antes de poder recuperarse; que los adictos tienen personalidades adictivas, e incluso, que sólo un adicto rehabilitado puede abordar y tratar la adicción desde un marco terapéutico. Todas estas ideas generalizadas sobre la adicción, según Dodes (2002), son una ficción: es la sustancia - o el acto- la que tiene el poder de convertir a alguien en un adicto.

Khantzian y Albanese (2008) elaboran la hipótesis de la automedicación-selfmedication hypothesis (SMH por sus siglas en inglés)
- estableciendo que el uso de drogas es un esfuerzo para buscar un alivio a un sufrimiento emocional. Establecen que el uso y/o abuso de sustancias - lícitas o ilícitas- tiene mayor prevalencia en personas que tienen un déficit en las funciones de autocuidado. Concluyen que las personas con estos déficits de auto cuidado son más propensas a la adicción (Dodes, 2010).

La hipótesis de automedicación los lleva a afirmar que la elección de una persona de consumir drogas está determinada por la habilidad de la sustancia para tratar su dolor emocional más profundo. Es decir, que la preferencia por una droga supone "algún grado de especificidad psicofarmacológica” (González-Guerras, 2008, p. 148). Para los autores, hay ganancia psicológica en la perpetuación del sufrimiento por la adicción, siendo ésta una forma repetitiva del individuo para alcanzar un sentido de control, incluso si para alcanzarlo se necesita de sufrimiento (Dodes, 2010). Esto se relaciona con el planteamiento de Freud (1930) de la droga como un "quita penas".

Al igual que Khantzian y Albanese (2008), Miller (1994) sostiene que las adicciones son una forma de automedicar un dolor psíquico como "una forma autodestructiva y desesperada de suprimir depresiones y traumas conscientes e inconscientes" (p. 753). Autores como Weissman, Slobetz, Prusoff, Mezrit y Howard, (1976) afirman que la depresión es un estado común en los consumidores de sustancias toxicas y sugieren que el duelo patológico, el trauma psíquico y la depresión clínica juegan un papel importante en la adicción a una sustancia.

Por otra parte, Khantzian y Wilson (1992) establecen que la drogadicción representa experiencias afectivas disfuncionales originadas en la niñez y, por lo tanto, "la adicción no implica únicamente una automedicación, sino que simultáneamente proporciona el sufrimiento psicofísico implícito [...] como un medio para controlar aquello que ha sido abrumador, desorganizado y desconcertante en la forma de afecto 
defensivo" (p. 275). Igualmente, Modell (1984) establece que la adicción es una forma de lidiar con el estrés post-traumático - sin importar si su naturaleza es infantil, crónica o aguda- y afirma que la compulsión a la repetición está íntimamente relacionada con el trauma para poder abarcar el ciclo repetitivo del dolor adictivo y la angustia. Khantzian y Wilson (1992) afirman que la repetición es una fuerza mayor en la adicción y se centran en la repetición del sufrimiento adictivo como un ritual diseñado para reforzar la fantasía de que el sujeto puede dominar sus experiencias afectivas tóxicas y establecen que este patrón es el resultado de interacciones materno-infantiles disruptivas. Así, entonces, según Miller (1994), la adicción tiene una naturaleza dual: por un lado, se percibe como una forma de automedicación para soportar aquello que es insoportable y, por otro lado, se percibe como "una compulsión a repetir una disforia intolerable resultado de la adicción y alimentada por una determinación insaciable por dominar aquello que ha sido destructivo y que se encuentra cruelmente fuera de su control" (p. 755).

A diferencia de autores de orientación lacaniana como Réquiz (2000) y Salamone (2011) quienes se oponen a los tratamientos que apuntan a la identificación del sujeto con el significante "adicto", Schoen (2009), en consonancia con el mainstream del psicoanálisis anglosajón, destaca la efectividad de esa identificación. Schoen es un analista de orientación jungiana y afirma que sólo la perspectiva jungiana de la psique puede explicar -en términos psicodinámicos- la complejidad y los aspectos únicos de la adicción y su respectiva rehabilitación (citado en Addenbrooke, 2010).

En su libro, Schoen (2009) afirma que hay poco conocimiento de la población general sobre el interés y el rol que Jung jugó en la creación de A.A. Para esta orientación, el alcoholismo, por ejemplo, es "la sed espiritual de nuestro ser por la totalidad" (citado por Addenbrooke, 2010 , p. 133) y la adicción surge por la baja au- toestima que produce un "yo falso en el individuo" (p. 133). Para este autor, la adicción es una fuerza malévola, "no susceptible a la razón o a los tipos de tratamiento, como medicamentos, análisis, o enfoques cognitivos, aplicados a otras formas de enfermedad mental" (citado en Addenbrooke, 2010, p. 134).

Si la adicción no es tratada, ésta convierte la vida del sujeto adicto en una tragedia $\mathrm{y}$, para reafirmar esta idea, utiliza metáforas e imágenes de mitos y cuentos de hadas para ilustrar la fuerza transpersonal de la adicción a lo que él llama "la sombra arquetípica" o "el mal arquetípico" (Addenbrooke, 2010). Igualmente, Schoen argumenta que sólo una fuerza de igual magnitud y potencia puede neutralizar la adicción y, así, enfatiza que Jung hizo bien al enviar a un paciente suyo "en una búsqueda de un ambiente donde pudiera vivir una experiencia de conversión, la cual, según Jung, podría ofrecer una vía para eliminar su alcoholismo" (citado en Addenbrooke, 2010, p. 134).

Según Schoen (2009), la adicción tiene todo un proceso evolutivo: en primer lugar, surge el uso de alguna sustancia tóxica $-\mathrm{u}$ objeto que en un principio no genera daño; luego, surge el abuso -o mal uso- de las mismas que, en efecto, sí provoca daño al sujeto en la medida en que esta actividad esté bajo "control"; y, finalmente, surge la adicción, caracterizada por la incapacidad del individuo de controlar lo que sucede en su ambiente y a su alrededor (Addenbrooke, 2010). Ésta última idea es coherente con Goldin (2014), quien argumenta que la adicción es "una falta de elección, una incapacidad para controlar el ambiente, un estado anti-simbólico que moviliza al sujeto de un modo narrativo de ser a un modo condicionado de ser o modo de feedback sintomático que se basa en gran medida en un refuerzo positivo y negativo y tiende a reducir los horizontes temporales" (p. 247). Schoen (2009) al respecto supone que la adicción es un fenómeno destructivo que sólo puede conocer su fin con un punto de transición radical, 
haciendo especial énfasis en grupos como A.A. para su rehabilitación (Addenbrooke, 2010).

Con la creación de A.A. se logró realizar una generalización sobre la adicción: 1) Cualquier persona puede llegar a ser adicto. 2) Una vez la adicción sienta sus bases, todos los pacientes se parecen. 3) La recuperación siempre es factible. Con estas generalizaciones se concluyó que la adicción como condición que afecta a un sujeto es una enfermedad (Fine y Juni, 2001).

Según Fine y Juni (2001), la adicción "implica una enfermedad consistente en términos de síntomas, patología, curso y órgano afectado" (p. 296), en donde el ego es el locus especifico de la enfermedad y su propósito es mantener la salud mental del sujeto y los síntomas son vistos como indicadores de procesos de atrofia del yo y de regresión. En su artículo, los autores presentan viñetas clínicas de las que concluyen que, sin importar las diferencias de status socioeconómico y cultural, "los sujetos mostraban fenómenos idénticos desde una perspectiva psicológica: negación, proyección y rabia” (p. 297). La negación y la proyección son mecanismos de defensa del yo ampliamente trabajados desde el psicoanálisis freudiano y annafreudiano.

Según Kahn (2002), la negación es "la manipulación mental de un hecho externo" (p. 148) que implica la protección contra la angustia por medio de una percepción errónea -0 ausente- de algún hecho que está más allá de los sentimientos y pensamientos propios; igualmente, en la negación, el ambiente exterior y la realidad interna son "reinterpretados para adaptarse a las necesidades defensivas del ego, mostrando una imagen que es incongruente con los hechos" (Fine y Juni, 2001, p. 299). La proyección, por su parte, manipula tanto la percepción interna como externa, protegiendo al individuo de la angustia por medio de la represión de un sentimiento y la percepción errónea de que es el otro quien experimenta ese sentimiento (Kahn, 2002).
En la proyección, el ego culpa al otro por la situación complicada que se está experimentando. En la adicción, el yo fluctúa constantemente entre estos dos mecanismos de defensa, "con opciones más extremas de una negación total de la realidad o con atribuciones erróneas masivas de culpa hacia otros" (Fine y Juni, 2001, p. 300). Los autores concluyen que la adicción es "el uso compulsivo de sustancias psicoactivas [...] caracterizado por el desarrollo de un síndrome psiquiátrico predecible" (p. 300 ), el cual usurpa el funcionamiento del yo y debilita las funciones preexistentes del yo.

Desde la teoría de las relaciones objetales, la naturaleza de los conflictos mentales se sitúa en los conflictos psíquicos, más que en los déficits. La naturaleza de las interacciones tempranas entre el bebé y su ambiente -generalmente representado por la figura maternajuega una importante influencia en el desarrollo de las relaciones entre la persona y los objetos -otras personas, seres animados o incluso seres inanimados como la sustancia- (Acuña, 2009). Así, por ejemplo, McDougall (1974) afirmó que los sujetos adictos tienen una falta de representación mental de un objeto bueno y deciden utilizar la droga a forma de compensar esa falta. Igualmente, sostenía que las adicciones son enfermedades psicosomáticas siendo éstas "todos los casos de daño físico o enfermedad en la que existen factores psicológicos desempeñando un rol importante" (Acuña, 2009, p. 7). Por lo tanto, una enfermedad psicosomática incluye la adicción al trabajo, al juego, al sexo, a la ingesta excesiva de alimentos, etc. Por otra parte, Krystal (1978) concibe que en la adicción la realidad psíquica se encuentra dominada por una experiencia de ambivalencia originada en un principio a una imago paterna para después extenderse a otros objetos -otras personas o incluso la misma droga (Acuña, 2009).

En relación al tóxico, el sujeto actúa tanto en las funciones de fusión como las de separación con respecto a la madre pues la adicción no sólo consiste en consumir sustancias psicoactivas, 
sino también en estar privado de sus efectos: "mientras el adicto añora su amado objeto, también le teme" (Krystal, 1978, p. 226). Debido a la ambivalencia de la relación, una completa unión con el objeto resulta amenazante debido a los sentimientos de odio, envidia y temores de salir herido, entre otros. Como resultado de la ambivalencia hacia la madre, el sujeto no puede introyectar su imagen y esta dificultad en internalizar la figura de la madre lleva a una falta de autocuidado, característica esencial de muchos sujetos adictos (Krystal, 1978).

\section{Una perspectiva del goce en la adicción}

Ahora veremos lo que distingue netamente la orientación por lo real en el abordaje de las adicciones. Se trata de un abandono en lo teórico de la pretensión de establecer universales a partir de los fenómenos particulares, acompañado de un acento puesto en el uso singular que cada sujeto hace del tóxico, extrayendo las enseñanzas que cada caso produce a partir de una reflexión permanente sobre la experiencia clínica. Esto, sin embargo, desemboca en un principio: en general el tóxico separa al sujeto del goce fálico y de esta manera lo aleja del sufrimiento concomitante con las dificultades del deseo, lo cual es consecuente con los avances freudianos y lacanianos sobre el tema.

La "orientación por lo real" es el término que utiliza Miller (2013) como el fenómeno dentro del psicoanálisis que pretende indagar y analizar de qué goza un sujeto y cómo lo hace y esto es una premisa fundamental para la práctica psicoanalítica. Lacan diferencia las nociones de placer y goce, afirmando que "el goce reside en el intento permanente de exceder los límites del principio del placer [...] es causa de sufrimiento, pero el sufrimiento no erradica nunca por completo la búsqueda del goce" (Roudinesco y Plon, 2008, p. 415). El psicoanalista se interesa especialmente en la relación que existe entre el sujeto y la sustancia tóxica (Salamone, 2011, p.54 y Réquiz, 2000, p. 48). Esto tiene una relación muy estrecha con el consumismo y con las prác- ticas propias del capitalismo contemporáneo. Réquiz afirma que la droga, como objeto, está también sujeta a las leyes del mercado, siendo especialmente lucrativa. Lacan (1970/2002) utilizó el término "letosas" para referirse a aquellos objetos de goce que el mercado produce, concepto que desarrolló en sus trabajos sobre la globalización del discurso capitalista. Según Karothy (2002), las letosas son "aspiradoras del deseo y prometedoras del goce" (citado por López, 2007, p. 34) y pueden extenderse infinitamente "desde creencias efusivas como la religión, hasta hábitos promovidos por el desarrollo de la tecnología digital, colocando los tóxicos químicos como una forma entre otras de adicción" (López, 2007, p. 34).

Desde el punto de vista de la "orientación por lo real" (Salamone, 2011), el psicoanalista escucha atentamente el discurso del sujeto, intentando capturar las nociones de goce y de sufrimiento del mismo, para así analizar cómo este sufrimiento puede presentarse como un significante para después convertirse en síntoma. En el paradigma freudiano, el síntoma implica que un significante aparece en el lugar de otro reprimido y, en general, en el síntoma clásico, cuando se habla de síntoma, lo que se pone en juego es un goce fálico. En el ámbito de las adicciones, por su parte, el vínculo entre sujeto y sustancia no se rompe porque hay un significante reprimido, sino que hace una ruptura de la relación entre sujeto y lenguaje.

Lacan (1975), para referirse a las sustancias tóxicas, retoma una metáfora del significante fálico tomado del caso Juanito: "no hay ninguna otra definición que la droga que ésta, es lo que permite romper el casamiento con la cosita de hacer pipí" (citado por Salamone, 2011, p. 55; Réquiz, 2000, p. 24 y López, 2007, p. $65)$. De esta afirmación se puede concluir que la droga es lo que permite romper la relación del sujeto con el falo, es decir, es una relación de quiebre (Tarrab, 1998, p. 38; Salamone, 2011, p 55). La consecuencia de esta ruptura con la dimensión fálica es llamada por Miller (1989) 
como "el problema sexual" (Réquiz, 2000, p. 24), tesis sostenida por la definición mencionada anteriormente de Lacan sobre la droga. El falo, cumpliendo una función simbólica, ordena el goce y su rechazo mediante la droga conlleva al rechazo del inconsciente y a una experiencia de goce sin Otro. En el problema sexual -cuando se rompe el matrimonio con el falo- el sujeto huye de los efectos de la castración y evita encontrarse con el falo para enfrentarse con la sexualidad.

En la adicción, es importante determinar cuál es el tipo de goce que permite al sujeto escapar de la castración y mantenerse con su objeto de consumo; el rechazo del falo acerca al individuo a un goce del cuerpo en solitario, que eventualmente conlleva al autoerotismo. Ahora bien, la medicina contemporánea reconoce la dependencia a un goce en la droga y a esto lo llama "adicción física", la cual "es el regreso sobre el cuerpo de un goce mortificante que tiene entre sus fines separar el cuerpo del matrimonio con el falo del que habla Lacan" (Réquiz, 2000, p. 25). Esa indicación de Lacan muestra a su vez cómo el sujeto adicto usa objetos para escapar de la relación con el falo.

Desde la teoría inicial de Freud, el falo sirve para inscribirse inconscientemente como hombre o como mujer y, dentro de las toxicomanías, el falo afecta al individuo en dos áreas distintas: 1) El sexo y la relación con el Otro sexuado que pasa por el falo y 2) La identidad sexual por la relación con el falo. Una de las consecuencias de esto es que el goce sexual "deriva hacia el autoerotismo en un proceso paralelo al alejamiento del Otro" (Réquiz, 2000, p. 26). Así, entonces, se ve cómo algunas relaciones de pareja en donde al menos uno de los individuos está ligado a una adicción, se rompen, pues la pareja es sustituida por la droga. Ruiz (2013) presenta una viñeta clínica de una paciente toxicómana en la que muestra cómo la droga pasa a ser la pareja de la paciente (su partenaire), siendo un objeto que sustituye la relación con los otros.
En los casos de sustancias que hacen una ruptura con el falo, Salamone (2011) afirma que la función fálica queda en suspenso, pues en la droga opera como una salida frente a una sexualidad que lo atormenta. Cuando el sujeto hace una ruptura con el falo mediante la intoxicación, lo que hace realmente es cerrar momentáneamente la problemática que la sexualidad implica (Tarrab, 1998). Sin embargo, no todas las drogas implican una ruptura con el falo. Mi1ler (2005) afirma que el alcoholismo no es una toxicomanía caracterizada por la ruptura con el falo y pone como evidencia casos en los que el alcoholismo está relacionado con el acto sexual: individuos que, para poder tener una relación sexual, necesitan del alcohol.

En la definición mencionada de Lacan sobre la droga, nótese que no es una definición de la adicción, sino de la droga como tal. Para el psicoanálisis, el interés está puesto, más que en la toxicomanía o la adicción, en la relación que establece el sujeto con la droga. Réquiz (2000) afirma que, en la práctica psicoanalítica, el interés en el ámbito de las adicciones no es que el sujeto deje de consumir, que es sin duda algo deseable, sino reintroducir al Otro en las relaciones del sujeto. Salamone (2011) considera que la sustancia rompe el vínculo con el lenguaje, dejando de lado la relación con el Otro, obteniendo un goce autoerótico sin necesidad del Otro. Para Freud, el autoerotismo implica la presencia de un objeto -aunque sea una representación mental- incluso si es el mismo cuerpo el que brinda la satisfacción. Se concluye entonces que el autoerotismo siempre implica un objeto. Para Freud, la masturbación, la cual ligaba estrechamente con las adicciones, es un acto autoerótico. Según Réquiz (2000), el problema yace en cómo entender el autoerotismo al que el sujeto se abandona en la adicción; igualmente, comenta que, en la adicción, el sujeto siempre está en busca de un goce que se escapa de la regulación y entonces se evidencia una falta de moral y una incapacidad para seguir las normas y leyes por ir en busca de un goce. La característica más representativa del goce es 
la repetición y, siendo así, la droga es un objeto de la pulsión (p. 47).

Réquiz (2000) plantea y critica que las psicoterapias tradicionales que tratan el problema de la adicción se centran en la detención del consumo y con eso determinan el éxito del tratamiento. Igualmente, plantea que estos tratamientos operan desde la identificación, para buscar "nuevos modelos de conducta que ayuden en el manejo del objeto, de las circunstancias [...], etc." (p. 15).

Desde el psicoanálisis, por el contrario, la cuestión no es únicamente detener el consumo, sino modificar las razones subjetivas involucradas en la adicción. Incluso, según Réquiz, la droga siempre cumple funciones en la economía mental del sujeto que éste puede descubrir en un tratamiento psicoanalítico para su beneficio. En las psicoterapias tradicionales, el éxito terapéutico perdura siempre y cuando se mantenga la identificación con el significante amo de la institución. La identificación consistiría en decir: "soy obeso", "soy depresivo", "soy alcohólico", etc., las cuales dejan por fuera el verdadero significante amo que está en juego para los sujetos. Desde la perspectiva de Salamone (2011), los tratamientos por la identificación son perjudiciales porque generan "una consistencia a la relación del sujeto con el tóxico" (p. 44). Cuando un sujeto dice "soy alcohólico" o "soy adicto", se está identificando con la sustancia y, desde el paradigma psicoanalítico, se plantea que el individuo tiene un problema con cierta sustancia cuya causa de consumo yace en el inconsciente. En un tratamiento psicoanalítico, se cuestiona cómo se manifiesta el goce de un sujeto y cómo se pueden desarticular, buscando dislocar la consistencia existente entre el sujeto y el tóxico.

Los tratamientos de rehabilitación que se centran en la identificación parten de la indeterminación significante del sujeto. Según Lacan, la indeterminación en el individuo surge como un efecto de la estructura propia del sistema significante.
"Si el Otro no me puede decir quién soy, luego no me queda otra alternativa sino acogerme a las identificaciones que ese mismo Otro me ofrece y sobre eso voy a construir un ser. Es el paso de la identificación a la identidad, pero [...] el ser no sería más que un trozo del Otro que incorporo como propio en el lugar de algo que falta” (Réquiz, 2000, p. 16).

Instituciones como Alcohólicos Anónimos (A.A.) o Narcóticos Anónimos (N.A.) basan su trabajo desde la premisa de la identificación y esta identificación postula básicamente que se debe hacer propio algo externo; de igual manera, los grupos como A.A. o N.A. piden una renuncia a ese objeto y en el proceso de la abstinencia proponen una identificación para afirmarse como adictos $-\mathrm{O}$ alcohólicos- $\mathrm{y}$, así, no volver al consumo. Por medio de esta identificación, se busca la detención de la adicción, reemplazando un objeto por un significante. Ahora bien, de la relación del individuo con la droga, es necesario especificar que es una relación directa en donde la palabra queda por fuera; con la droga, el sujeto organiza su vida y "despoja al objeto de significaciones hasta dejarlo [...] vaciado de sentido" (Réquiz, 2000, p. 20), funcionando como un límite significante.

En A.A. o N.A., después de la identificación del sujeto como adicto, viene la abstinencia en un postulado que afirma "soy eso que el objeto define, pero soy sin su presencia física" (Réquiz, 2001, p.20). Desde el psicoanálisis, sin embargo, se trata de abrir al sujeto a "la posibilidad de dialectizar la rigidez [de las identificaciones], y soltar con ello [...] las amarras $[\ldots]$ que los significantes escriben para cada sujeto" (Réquiz, 2000, p. 21).

\section{Conclusiones}

En este trabajo se presentó una revisión de literatura sobre la perspectiva del psicoanálisis con respecto a las adicciones, haciendo un recorrido desde la teoría inicial de Sigmund Freud y, posteriormente, Jacques Lacan, incluyendo algunos trabajos posteriores de psicoana- 
listas anglosajones, con la finalidad de hallar lo que distingue la orientación por lo real de otras aproximaciones psicoanalíticas con respecto a esta problemática. De ello se deriva el acento que la elaboración psicoanalítica pone sobre la vertiente ética de asunto de las adicciones, que se fundamenta en la existencia del problema del uso que hace un sujeto en su especificidad del tóxico.

Es pertinente mencionar que, si bien Freud elaboró algunas ideas sobre las adicciones, no elaboró una teoría sobre la toxicomanía como tal. Al comenzar su teoría psicoanalítica sobre las adicciones, la percepción de Freud sobre este fenómeno yacía en el campo de la sexualidad infantil, afirmando que la masturbación es la primera adicción de la cual surgen las demás adicciones. Así mismo, y si bien no lo menciona explícitamente en su elaboración teórica sobre la toxicomanía, le brinda un importante rol a la influencia social y cultural como elementos esenciales en la formación de una adicción. En El Malestar en la Cultura (1930), Freud explica que gran parte de estos "dolores" surgen por la búsqueda incesante del ser humano por la felicidad y, más específicamente, por evitar el displacer, siendo la sustancia un "quita penas" (p. 75).

Tanto la influencia como el contexto cultural y social juegan un importante rol en la formación de una adicción, idea es respaldada por Le Poulichet (1996) cuando afirma que "la pérdida de los valores, la abdicación de los progenitores cada vez más sobrepasados por la rapidez y la violencia de la evolución socioeconómica empujan [...] a los sujetos hacia la toxicomanía" (p. 23). Freud afirma que no toda persona que consuma una sustancia desarrolla una adicción a ésta y plantea que en el tratamiento de esta afección no se debe privar al sujeto de su objeto de deseo únicamente, sino también aumentar las satisfacciones del individuo.

Lacan, por su parte, inicia sus primeras ideas sobre la adicción a partir del complejo de destete, afirmando que la adicción surge a raíz de un trauma no elaborado. Así mismo, no considera el consumo de sustancias como un recurso o como una vía para conocer el inconsciente, pues la sustancia es un recurso cuyo fin es evitar una verdad intolerable que sólo se dice en la palabra. Posteriormente, Lacan (1975) desarrolla la relación del sujeto con la sustancia, afirmando que ésta le permite hacer una ruptura con el falo.

Según Miller (1989), esta ruptura del sujeto con el falo recibe el nombre de "problema sexual" en donde el individuo huye de los efectos de la castración y evita encontrarse con el falo para enfrentarse con la sexualidad. Salamone (2011) afirma que la sustancia rompe el vínculo con el lenguaje y, al hacer esto, se deja de lado la relación con el Otro, obteniendo así un goce sin necesidad del Otro y, al hacer esto, el goce se ubica en el autoerotismo.

Desde otro campo del psicoanálisis, que es la vertiente de la teoría de las relaciones objetales, la naturaleza de las interacciones tempranas entre el bebé y su ambiente generalmente representado por la figura materna- juegan una importante influencia en el desarrollo de las relaciones entre la persona y los objetos -otras personas, seres animados o incluso seres inanimados como la propia sustancia (Acuña, 2009). Así, entonces, la droga es utilizada como forma de compensar una falta de representación mental de un objeto bueno e idealizado.

En cuanto a la necesidad de determinar la adicción como una enfermedad, y a los obstáculos existentes para la convergencia entre el psicoanálisis y la medicina para el entendimiento del fenómeno de la adicción, se puede afirmar que dichos obstáculos se relacionan con la forma en la que se ha caracterizado el fenómeno adictivo desde ambas aproximaciones. En general, la visión psicoanalítica del fenómeno de la adicción acepta la premisa según la cual la sustancia siempre cumple una función 
en la economía mental y libidinal del individuo y hace un fuerte énfasis en aumentar las satisfacciones del sujeto y en indagar por las causas subyacentes al consumo, más no considera que la abstinencia es fundamental para el cese del consumo y la recuperación de la adicción.

Desde finales del siglo XIX hasta nuestros días, los psicoanalistas han venido investigando y elaborando comprensiones sobre el fenómeno de la adicción y sobre sus fundamentos clínicos. Con el auge contemporáneo del consumo de sustancias, el aumento en la diversidad de sustancias psicoactivas producidas y consumidas, y los cuadros clínicos derivados y asociados a ese consumo, se hace evidente y necesario que continuará siendo un campo de investigación y publicación que buscará brindar nuevos entendimientos del fenómeno de la adicción y de sus tratamientos posibles.

\section{Referencias}

Abraham, K. (1908). Letter from Karl Abraham to Sigmund Freud, August 21, 1908. The Complete Correspondence of Sigmund Freud and Karl Abraham 1907-1925, 5657.

Acuña, A. (2009) Una aproximación psicoanalítica contemporánea al entendimiento de las adicciones. Recuperado de http:// www.schilesaludmental.cl/2009_enero_congreso/talleres/DR.\%20ACŪNA/ Dr_Acuna.pdf

Addenbrooke, M. (2010). The War of the Gods in Addiction: C.G. Jung, Alcoholics Anonymous and Archetypal Evil. Journal of Analytical Psychology, 55,134-135.

Balint, M. (1979). La falta básica. Barcelona: Paidós.

Barrios, J. (2011) Pijamasurf. ¿Cuánto nos tocaría a cada quien si se repartiera todo el dinero del mundo? Recuperado de http://
pijamasurf.com/2011/10/\%C2\%BFcuantonos-tocaria-a-cada-quien-si-se-repartieratodo-el-dinero-del-mundo/

Bateman, A., y Holmes, J. (1995) Introduction to psychoanalysis. Contemporary theory and practice. London and New York: Routledge.

Bateman, A., Brown, D., y Pedder, J. (2000). Introduction to psychotherapy. An outline of psychodynamic principles and practice. London: Routledge

Bergeret, J. (1983). Le toxicomane et sa psychothérapie. Cahiers Critiques de Thérapie Familiale et de Pratique des Réseaux. Paris: Universitaires.

Bleuler, E. (1911). Alkohol und Neurosen. Jahrbuch für psychoanalytische und psychopathologische Forschungen, 3, 848-852.

Champion, L.A., Goodall, G.M., y Rutter, M. (1995). Behavioural problems in childhood and stressors in early adult life: a 20 years follow-up of London school children. Psychological Medicine, 25, 231-246.

Deutsch, H. (1926). Occult Processes Ocurring in Psychoanalysis. Imago, 12, 418-433.

Dodes, L.M. (1990). Addiction, helplessness and narcissistic rage. Psychoanalytic Quarterly, 59, 398-419.

Dodes, L.M. (2010). Understanding Addiction as Self Medication: Finding Hope behind the Pain. By Edward Khantzian and Mark Albanese. Lanham, MD: Rowman and Littlefield, 2008. [Review]. Psychoanalytic Quarterly, 79(2), 582-585.

Epstein, S. (1994). Integration of the cognitive and psychodynamic unconscious. American Psychologist, 49, 709-724. 
Fava, C. (2013). Se le escapan de las manos. En L.D. Salamone (Ed.). Todos adictos en la agitación de lo real.Pharmakon 13. (pp.101-104). Buenos Aires: Grama. Ferenczi, S. (1911). Über die Rolle der Homosexualität in der Pathogenese der Paranoia. Jahrbuch für psychoanalytische und psychopathologische Forschungen, $3,101-119$.

Fine, J \& Juni, S. (2001). Ego Atrophy in Substance Abuse: Addiction from a Socio-Cultural Perspective. American Journal of Psychoanalysis, 61, 293-304.

Freud, S. (1884/1980). Sobre la cocaína. En R. Byck (Ed.). Escritos sobre la cocaina. (pp. 7-410) Barcelona: Anagrama.

Freud, S. (1898/2010) La sexualidad en la etiología de la neurosis. En J. Strachey (Ed). Obras completas. (Vol. 3, pp. 251-264). Buenos Aires, Argentina: Amorrortu.

Freud, S. (1905/1997). El chiste y su relación con lo inconsciente. En J. Strachey (Ed.). Obras completas. (Vol. 8, pp. 1-237). Buenos Aires: Amorrortu.

Freud, S. (1908/2010). La moral sexual cultural y la nerviosidad moderna. En J. Strachey (Ed.). Obras completas. (Vol. 9, pp. 159170). Buenos Aires: Amorrortu.

Freud, S. (1910/1961). The future prospects of psycho-analytic therapy. En: J. Strachey (Ed.). The Standard Edition of the Complete Psychological Works of Sigmund Freud. (Vol. 2, pp. 144-151). London: Hogarth Press.

Freud, S. (1915/1996). Pulsiones y destinos de pulsión. En J. Strachey (Ed). Obras completas. (Vol. 14, pp. 105-134). Buenos Aires: Amorrortu.
Freud, S. (1927/2010). El humor. En J. Strachey (Ed.). Obras completas. (Vol. 21, pp. 153163). Buenos Aires: Amorrortu.

Freud, S. (1928/2010). Dostoyevski y el parricidio. En J. Strachey (Ed.). Obras completas. (Vol. 21, pp. 171-194). Buenos Aires: Amorrortu.

Freud, S. (1930/2010). El malestar en la cultura. En En J. Strachey (Ed.). Obras completas. (Vol. 21, pp. 57-140). Buenos Aires: Amorrortu.

Freud, S. (1937). Construcciones en el análisis. En J. Strachey (Ed.). Obras completas. (Vol. 23, pp. 255-270). Buenos Aires: Amorrortu.

Gavlovski, J. (2011). Cuando la droga falla. Caracas: Pomaire.

Gittelson, R. (1952). The Emotional Position of the Analyst in the Psychoanalytic Situation. International Journal of Psychoanalysis, 33, 1-10.

Glover, E. (1928). The etiology of alcoholism. En Glover, E. (Ed.). On the early development of mind (pp. 81-90). New York: International University Press.

Goldin, D. (2014). Addiction and Temporal Bandwidth. International Journal of Psychoanalytic Self Psychology, 9(3), 246-262.

González-Guerras, J. (2008). Psicoanálisis y Toxicomanías. Clínica e Investigación Relacional, 2, 146-164.

Gottdiene, W. (2010). Understanding Addiction as Self Medication: Finding Hope Behind the Pain. Journal of the American Psychoanalytic Association, 58, 10281032. 
Gutiérrez-Peláez, M. (2012). Confusión de lenguas. Un retorno a Sandor Ferenczi. Buenos Aires,: Eudem.

Heimann, P. (1949/1950). On Countertransference. In: Collected Papers: About children and children no longer. London: The New Library of Psychoanalysis.

Heyman, G. M. (2009). Addiction: A disorder of choice. Cambridge, MA: Harvard University Press.

Iriarte, M. (2009). Un lazo intoxicado por la ética del soltero. En: J. Miller (Ed.). El lazo social intoxicado. Pharmakon 11. (pp. 31-34). Buenos Aires: Grama.

Jaffe, L. (2002). In-Patient Treatment of Substance Abuse. International Journal of Psychoanalysis, 83, 253-256.

Jellinek, E. (1960). The Disease Concept of Alcoholism. New Haven: Hillhouse.

Johnson, B. (1999). Three perspectives on addiction. Journal of the American Psychoanalytic Association, 47(3), 791815.

Josson, J. M. (2013). La función de la droga. En L. D.Salamone (Ed.). Todos adictos en la agitación de lo real. Pharmakon 13. (pp.121-127). Buenos Aires: Grama.

Kahn, M. (2002). Freud básico: psicoanálisis para el siglo XXI. Buenos Aires: Emecé.

Kandel, E. R. (1999) Biology and the future of psychoanalysis: a new intellectual framework for psychiatry revisited. American Journal of Psychiatry, 156, 505-524.

Karothy, R. (2002) Contexto en psicoanálisis $N^{\circ}$ 6: las adicciones. Buenos Aires: Lazos.
Kernberg, O. (1975). Borderline conditions and pathological narcissism. New York: Aronson.

Khantzian, E. J., y Mack, J. E. (1983). Selfpreservation and the care of the self. Ego instincts reconsidered. Psychoanalytic Study of the Child, 38, 209-232.

Khantzian, E. (1985). The self-medication hypothesis of addictive disorders. American Journal of Psychiatry, 142, 1259-1264.

Khantzian, E., y Wilson, A. (1992). Substance Abuse Repetition and the Nature of Suffering. En: Wilson \& G. Gedo. (Eds.). Hierarchical Concepts in Psychoanalysis: Theory, Research and Practice. (pp.263283). New York: Guilford Press.

Khantzian, E. (2005). New Windows on Understanding Addictive Vulnerability: Commentary on Papers by Lisa Director and Noelle Burton. Psychoanalytic Dialogues, 15, 613-620.

Khantzian, E. J., \& Albanese, M. J. (2008). Understanding addiction as selfmedication: Finding hope behind the pain. Lanham, MD, US: Rowman \& Littlefield.Kihlstrom.

Kohut, H. (1971). The analysis of the self. New York: International University Press.

Krystal, H. (1978). Self-representation and the capacity for self-care. Annual of Psychoanalysis, 6, 209-246.

Lacan, J. (1959/1985). Subversión del sujeto y dialéctica del deseo en el inconsciente freudiano. Escritos II. Buenos Aires: Siglo Veintiuno.

Lacan, J. (1959/2015). El deseo y su interpretación. El seminario, Libro 6 . Buenos Aires: Paidós. 
Lacan, J. (1970/2002). El reverso del psicoanálisis. El seminario, Libro 17. Buenos Aires: Paidós.

Lacan, J. (1977). La familia. Buenos Aires: Argonauta.

Lacan, J. (1988/2002). Función y campo de la palabra y del lenguaje en psicoanálisis. Escritos I. Buenos Aires: Siglo Veintiuno.

LeDoux, J. E. (1992). Emotion as a memory: anatomical systems underlying indelible neural traces. In: Christianson, S-A. (Ed.). Handbook of emotion and memory. Hillsdale: Eribaum.

Le Poulichet, S. (1996). Toxicomanías y Psicoanálisis. Buenos Aires: Amorrortu.

Little, M. (1951). Counter-Transference and the Patient's Response to it. International Journal of Psychoanalysis, 32, 32-40.

López, C. (2006) La adicción a sustancias químicas: ¿Puede ser efectivo un abordaje psicoanalítico? Psykhe, 15, 67-77.

López, H. (2007) Las adicciones. Sus fundamentos clínicos. Buenos Aires: Lazos.

López, C. (2011) Adicción a sustancias químicas: ¿Enfermedad primaria o síntoma psicoanalítico? Revista de Psicología, 20, 41-60.

Malengreau, P. (2013) Apuntar al síntoma. En L.D. Salamone (Ed.). Todos adictos en la agitación de lo real. Pharmakon 13. (pp.8286), Buenos Aires, Argentina: Grama.

McDougall, J. (1974). The psychosoma and psychoanalytic process. International Review of Psychoanalysis, 1, 437-454.

Meltzer, D. (1974). Los estados sexuales de la mente. Buenos Aires: Kargieman.
Menéndez, E., Di Pardo, R. (2006). Alcoholismo: políticas e incongruencias del sector salud en México. Desacatos, Revista de Antropología Social, 20, 29-52.

Miller, J. (1994). Substance Abuse: The Role of Depression and Trauma: A Case Report. Journal of American Academy of Psychoanalysis, 22, 753-764.

Miller, J.A. (2008). El partenaire- sintoma. Cursos psicoanalíticos de J-A. Miller. Paris: Paidós.

Miller, J.A., (2013). El ultimísimo Lacan. Buenos Aires: Paidós.

Miller, W. R. y Rollnick, S. (1991). Motivational Interviewing: Preparing People to Change Addictive Behaviour. New York: Guilford Press.

Ministerio del interior y de justicia, Ministerio de la protección social y Dirección Nacional de Estupefacientes. (2008). Estudio Nacional del Consumo de Drogas en Colombia. Recuperado de http://www1.urosario.edu. co/correo/administracion/boletines/ODA/ documentos/ESTUDIO_NACIONAL_ SOBRE_DROGAS_COLOMBIA.pdf

Modell, A. (1984). Psychoanalysis in a New Context. New York: International Universities Press.

Morse, R. M., y Flavin, D. K. (1992). The definition of alcoholism. The Joint Committee of the National Council on Alcoholism and Drug Dependence and the American Society of Addiction Medicine to Study the Definition and Criteria for the Diagnosis of Alcoholism. Journal of American Medical Association, 268(8), 1012-1014.

Naparstek, F. (2014). Clínica de las adicciones. Encuentro Psicoanalítico. Asociación Lacaniana de Psicoanálisis (ALP). Chile. 
Rado, S. (1926). Los efectos psíquicos de los intoxicantes: un intento de desarrollar una teoría psicoanalítica de los deseos morbosos. En S. Rado(Ed.). Psicoanálisis de la conducta (pp. 33-48). Buenos Aires: Horm.

Rado, S. (1933). Psicoanálisis de la farmacotimia (Afición a las drogas). En S. Rado (Ed.). Psicoanálisis de la conducta (pp. 73-89). Buenos Aires, Argentina: Horm.

Réquiz, G. (2000). La perspectiva psicoanalítica de las adicciones. [En prensa]. Nueva Escuela Lacaniana, Cali, Colombia.

Rivers, C., y Shore, E. (1997). Substance abuse on campus. New York: Greenwood Press.

Rodríguez de la Sierra, L. (2012). Countertransference: Our difficulties in the treatment of substance abuse. British Psychoanalytical Society, 5, 76-92.

Rosenfeld, H.A. (1960). On Drug Addiction. International Journal of Psycho-Analysis, 41, 467-475.

Roudinesco, E., y Plon, M. (2008). Diccionario de psicoanálisis. Buenos Aires: Paidós.

Ruiz, S. M. (2013). El partenaire y la droga en un caso de una mujer toxicómana. En L. D. Salamone (Ed.). Todos adictos en la agitación de lo real. Pharmakon 13. (pp. 82-86), Buenos Aires: Grama.

Rutter, M. (1999). Psychosocial adversity and child psychopathology. British Journal of Psychiatry, 174, 480- 493.

Sachs, H. (1923). Zur Genese der Perversionen. Internationale Zeitschrift für Psychoanalyse, 9(2), 172-182.

Salomone, L. D. (2011). Cuando La droga falla. Caracas: Pomaire.
Sánchez, C. (2014). El Universal de 10mx. 10 países donde el consumo de marihuana es legal. Recuperado de http://de10.com.mx/ vivir-bien/2014/10/29/10-paises-dondeel-consumo-de-marihuana-es-legal

Sandler, J. (1976). Countertransference and role responsiveness. International Review of Psychoanalysis, 3, 43-47.

Silverman, M. (2005). The heart of addiction. Psychoanalytic Quarterly, 74, 912-917.

Southwick, S., y Satel, S. (1990) Exploring the meanings of substance abuse: an important dimension of early work with borderline patients. American Journal of Psychotherapy, 46, 61-67.

Strauss, L. (1996). Persecución y arte de escribir. Valencia: Alfons el Magnánim.

Tarrab, M. (1997/1998). Una Experiencia Vacía. En J. Miller (Ed.). Pharmakon 6/7. (pp. 37-42). Buenos Aires: Grama.

Underwood, G. (1996). Implicit Cognition. New York, USA: Oxford University Press.

Van der Kolk, B. (1996). The body keeps the score: Approaches to psychobiology of posttraumatic stress disorder. In: van der Kolk, B., Mc Farlane, A., y Weisaeth, L. (Ed.). Traumatic Stress. The effects of overwhelming experience on mind, body and society. New York and London: The Guilford Press.

Vitale, A. (2012). Lectura Lacaniana. Problemas actuales para el psicoanálisis: nuevos síntomas-prejuicios del psicoanalista-. Recuperado dehttp://www.lecturalacaniana.com.ar/doc1.php?doc $=347$

Weissman, M., Slobetz, F., Prusoff, B., Mezritz, M., y Howard, P. (1976). Clinical depression among narcotic addicts maintained 
on methadone in the community. American Journal of Psyquiatry, 133, 1434-1438.

Whitman-Raymond, R.G. (1988). Pathological gambling as a defense against loss. Journal of Gambling Behavior, 4(2), 100-108.

Whitam-Raymond, R.G. (2005). Building the Nest: Reworking Adolescent Development in Adult Recovery from Addiction. American Journal of Psychoanalysis, 69, 136-149.

Winnicott, D.W. (1965). The maturational processes and the facilitating environment. London: The Hogarth Press
Winnicott, D. (1979). Realidady juego. Barcelona: Gedisa.

Wurmser, L. (1974). Psychoanalytic considerations of the etiology of compulsive drug use. Journal of American Psychoanalytic Association, 22, 820-842.

Wurmser, L. (1985). Denial and split identity: timely issues in the psychoanalytic psychotherapy of compulsive drug users. Journal of Substance Abuse Treatment, 2, 89-96. 